\title{
Linx
}

Revue des linguistes de l'université Paris X Nanterre

$80 \mid 2020$

L'héritage de Jean Dubois et Françoise Dubois-Charlier

\section{Le rôle complexe de modérateur dans le débat politique Question Time, vers une logométrie de l'absence?}

The complex role of moderator in the political debate Question Time. Towards a logometrical analysis of "absence".

\section{Laurent Rouveyrol}

\section{OpenEdition}

Journals

Édition électronique

URL : http://journals.openedition.org/linx/6471

ISSN : 2118-9692

Éditeur

Presses universitaires de Paris Nanterre

Référence électronique

Laurent Rouveyrol, « Le rôle complexe de modérateur dans le débat politique Question Time, vers une logométrie de l'absence? », Linx [En ligne], 80 | 2020, mis en ligne le 30 juin 2020, consulté le 05 août 2020. URL : http://journals.openedition.org/linx/6471

Ce document a été généré automatiquement le 5 août 2020

Département de Sciences du langage, Université Paris Ouest 


\section{Le rôle complexe de modérateur dans le débat politique Question Time, vers une logométrie de l'absence?}

The complex role of moderator in the political debate Question Time. Towards a logometrical analysis of "absence".

\section{Laurent Rouveyrol}

\section{Introduction}

1 Cet article est tiré du chapitre 4 portant sur les journalistes de Question Time de la thèse soutenue en 2003 (Rouveyrol 2003a) et dirigée par Françoise Dubois-Charlier. Ce chapitre est celui qui a véritablement passionné Françoise alors qu'elle exprimait des réserves sur son intérêt a priori. J'ai insisté, arguant du fait que suite à une étude préalable faite pour un congrès à Cuba (Rouveyrol 2003b), j'avais entrevu des éléments intéressants, elle m'a alors invité à explorer la piste des journalistes. Cette anecdote est d'ailleurs très révélatrice de l'extrême bienveillance avec laquelle elle a accompagné la rédaction de la thèse pendant plusieurs mois. Jamais je n'ai eu à affronter une quelconque opposition, même si la syntacticienne très segmentale et rigoureuse qu'elle représentait en linguistique anglaise a dû sourciller plus d'une fois devant les outils et les phénomènes que je présentais en analyse de discours, aux contours nécessairement plus flous. Ses réactions étaient toujours positives, m'orientant sur telle ou telle piste sur le mode du conseil avisé, m'encourageant en prônant l'ouverture à tous les niveaux. Françoise Dubois-Charlier était chercheur dans l'âme, ne sacrifiant jamais rien aux données, ni au corpus. C'est essentiellement grâce à ce soutien sans faille que j'ai pu venir à bout de ce travail, à une époque où en tant que PRCE, je devais gérer plus de 1000 étudiants par an. Son efficacité bienveillante a toujours été une référence absolue 
pour moi, notamment quand j'ai eu l'occasion d'encadrer des travaux de recherche à mon tour.

Le discours des journalistes dans les débats politiques télévisés ne génère pas vraiment d'intérêt en soi. C'est parfaitement normal si l'on considère que le rôle consiste essentiellement à susciter la parole et à la modérer; par nature, le présentateur de débat est au moins a priori en retrait, voire inexistant. Pourtant il est aussi le meneur de jeu par lequel tout ou presque doit transiter. Il s'agit donc d'une instance énonciative complexe, voire paradoxale, et nous partirons du principe que cette tension entre effacement et gestion effective du débat est en soi une problématique à étudier.

3 Cet article a donc essentiellement pour ambition de caractériser le discours des journalistes menant les débats dans Question Time et se situe dans le cadre de la perspective logométrique, essentiellement comparatiste (Mayaffre 2005). L'objet est de caractériser mais aussi de quantifier les stratégies de discours auxquelles les présentateurs de l'émission ont recours. Le corpus sera constitué de deux émissions sur les six composant le corpus de thèse car deux journalistes différents sont à l'œuvre : Peter Sissons pour la première (1992) et David Dimbleby pour la seconde (1997). Le contexte politico-social est différent : 1992 correspond au lendemain de la 4 ème élection des conservateurs, 1997 est la date de la première élection de Tony Blair et marque le retour au pouvoir des Travaillistes. La question essentielle est donc la suivante : quelle stratégie de positionnement les deux journalistes vont-ils adopter dans le contexte général qui correspond à celui de l'émission qu'ils présentent?

\section{Le journaliste meneur de jeu de Question Time : une instance locutive complexe}

On rappellera pour commencer un certain nombre de caractéristiques essentielles ${ }^{1} \mathrm{du}$ débat hebdomadaire Question Time. Il est diffusé par la BBC depuis 1978 et constitue une véritable institution en Grande-Bretagne depuis ses débuts.

5 La « recette » est toujours la même, quelques invités constituent un panel : en principe deux politiciens de bords opposés sont invités simultanément ainsi que deux autres personnalités de la vie civile (hommes et femmes de médias, intellectuels, experts divers ou tout simplement star du show-business). Des questions concernant l'actualité de la semaine sont soumises à la production par le public présent sur le plateau et sont ensuite tirées au sort. Les invités s'expriment tour à tour à l'invite du journaliste, qui a donc pour mission dans ce cadre de susciter la parole, la modérer, réguler les débats.

6 Nous avons donc affaire à un «chef d'orchestre " qui reste également effacé, cela constitue en soi un paradoxe intéressant que n'ont pas manqué de relever certains chercheurs. Pour Kerbrat-Orecchioni (1987: 324), le présentateur

[...] abdique sur un autre front puisque son rôle est moins de parler que de susciter la parole de l'autre, auquel il laisse le soin de fournir l'essentiel de la matière conversationnelle. Pilote effacé du dialogue, initiateur vite condamné à un silence relatif, l'interviewer est à la fois dominant et dominé (ces deux composantes se dosant diversement selon les interviews).

Ghiglione et al. (1989: 64) parlent assez justement d'hyper-locuteur qui a, si besoin, la possibilité de rappeler les règles du jeu. Un débat est un cadre égalitaire a priori (au moins 
en théorie) pour les participants, mais les invités vont vouloir agir sur ce cadre pour tenter d'arriver à une position "dominante", la régulation de la rencontre est donc essentielle pour lui garantir une dimension éthique incontestable. En tant qu'homme ou femme de média, le journaliste présentateur de débat télévisé a aussi la mission de générer un produit qui satisfasse le spectateur-auditeur, consommateur indispensable (Kerbrat-Orecchioni, $1990: 120$ ).

On notera donc que cette double tension lui confère à la fois une position haute pour ce qui est de la conduite des débats et une position basse dans la participation effective au débat d'idées. On pourrait dire aussi que les journalistes-présentateurs sont symboliques de l'hétérogénéité constitutive du cadre, qui rejaillit sur eux et sur tous les rôles qu'ils doivent investir au fil du programme.

Cette ambiguïté générique transparaît clairement dans le rôle du présentateur : estil ou elle le meneur d'un débat, le héros adulé d'un talk-show, un arbitre, un conciliateur, un juge, le présentateur enthousiaste d'un jeu télévisé, l'hôte d'une conversation au cours d'un dîner, un manager ou un porte-parole. Parfois, le présentateur joue chacun de ces rôles, modifiant ainsi le rôle des autres participants et des destinataires. (Livingstone et Lunt, $1994: 56$, notre traduction).

On trouve parfois des représentations plus surprenantes tenant compte du caractère sacralisant lié aux médias: ces journalistes jouissent parfois d'une reconnaissance sociale exceptionnelle ainsi que d'une notoriété internationale. Livingstone et Lunt (1994: 60) proposent la perspective suivante :

$10 \mathrm{Au}$ lieu de rester en retrait de façon neutre, supervisant l'action, le présentateur joue un rôle central, comme le héros qui entreprend de résoudre un problème affectant le royaume (le public), et de restaurer l'ordre social (par des conseils, la compréhension et validation des expériences). Ainsi, il traverse à grands pas le royaume mythique (le studio), dans un périple placé sous le signe de la découverte, brandissant son épée (le micro). En chemin, le héros rencontre ceux qui peuvent prodiguer des conseils et offrir des informations, ainsi que ceux qui s'érigent en obstacle en posant des problèmes, sapant l'information et qui, finalement, sont bannis : "ceci ne nous mène nulle part". Ayant atteint son but : la compréhension d'un fait, une décision, une preuve, le héros s'en retourne triomphal dans la communauté qui en a tant besoin, et pour qui l'ordre social est finalement rétabli et célébré.

11 Enfin pour la CDA (Critical Discours Analysis), ce genre télévisuel correspond à 3 ordres de discours distincts : le discours médiatique, le discours du système politique et le discours de la vie ordinaire (Fairclough : 1995). Les journalistes ont donc pour mission de les articuler et de les faire articuler.

12 Les questions structurantes qui vont maintenant se poser sont donc les suivantes: comment le journaliste du débat va-t-il jouer son rôle et se positionner? Quel ordre va$\mathrm{t}$-il privilégier : va-t-il porter la parole du public et du téléspectateur-citoyen? Va-t-il se placer du côté de l'institution privilégiant alors les politiciens au pouvoir? Que nous révèlent les stratégies adoptées?

13 A titre d'illustration pour ces enjeux, nous donnerons deux exemples tirés des débats présidentiels américains de 2004. Jim Lehrer modère le premier débat entre GW. Bush j.r et John Kerry, voici comment s'ouvre le débat :

- Good evening, Mr. President, Senator Kerry. As determined by a coin toss, the first question goes to you, Senator Kerry. You have two minutes.

Do you believe you could do a better job than President Bush in preventing another 9/11type terrorist attack on the United States? 
- New question, Mr. President, two minutes.

Do you believe the election of Senator Kerry on November the 2nd would increase the chances of the U.S. being hit by another 9/11-type terrorist attack? au Président Bush et au Sénateur Kerry, ce qui les convoque à des positions non symétriques. Le principe de réalité est bien là : Bush est à ce moment un président des Etats-Unis certes en campagne et Kerry occupe effectivement un mandat de sénateur. La façon de poser la question est beaucoup plus parlante : la structure comparative ( $a$ better job than) place de fait Bush en tant que repère à partir duquel Kerry est sommé de se situer. «A better job » est également intéressant. Un comparatif ne présuppose pas nécessairement l'adjectif de référence (Jean est plus grand que Paul ne présuppose pas que Paul est grand), mais dans le cas d'un axiologique les choses sont plus floues: d'une certaine façon on entend tout de même «a good job ». La formulation de la question n'est pas neutre ; on aurait pu imaginer plus objectivement : « what would you do in order to prevent another 9-11 terrorist attack? ». Si l'on avait encore quelques doutes, la question suivante permet de les dissiper rapidement: "Would the election of Senator Kerry increase the chances of the US being hit by another terrorist attack?" Le journaliste énonce explicitement une perspective suivant laquelle Kerry (candidat à la Présidence des Etats Unis) représente un danger potentiel pour la sécurité du pays... On ne peut pas dire que la formulation des questions soit à son avantage, Kerry est dès le départ condamné à une douloureuse immanence. On voit donc bien ici que tout cadre égalitaire (comme un débat présidentiel) peut être nuancé en fonction des positionnements adoptés par les différents participants et particulièrement des journalistes devant organiser ces débats : de quel côté sont-ils/décident-ils d'être finalement?

Cette vision de la langue comme outil de positionnement (et pas seulement comme vecteur de transmission du sens) correspond très bien à celle que Fairclough (1995) donne de la « modalité » :

A clause which codes an event (ideationally) in terms of a particular type of process will also assess (interpersonally) the truth or probability of the proposition so encoded, and the relationship between producer and addressee(s). The concept of 'modality' is used in a very general way to cover features of texts which express speakers' and writers' attitudes towards themselves, towards their interlocutors and towards their subject-matter' (Fowler et alii, 1979: 200). Choices of pronouns, modal auxiliaries, speech acts, and many others, are included within modality.

Les marques linguistiques observées dans cette étude le seront donc dans cette perspective : elles émaneront de toutes les strates discursives et seront considérées comme la trace d'une forme de positionnement.

\section{Le discours des journalistes modérateurs de Question Time}

\subsection{Premières constatations quantitatives}

17 Comme nous l'avons dit en introduction, deux émissions serviront de base à l'étude. L'une date de 1992 et est présentée par Peter Sissons; l'autre date de 1997 et est présentée par David Dimbleby. Voici dans le tableau suivant les premières données quantitatives. 
Tableau $\mathrm{n}^{\circ} 1$. Premières données chiffrées interaction 1 / Interaction 2

\begin{tabular}{|c|c|c|}
\hline & $\begin{array}{l}\text { INTERACTION } 1 \\
\text { Peter SISSONS }\end{array}$ & $\begin{array}{l}\text { INTERACTION } 2 \\
\text { David DIMBLEBY }\end{array}$ \\
\hline Nombre d'occurrences total (tokens) ${ }^{2}$ : & 11432 & 9893 \\
\hline Discours du journaliste (tokens) & 1494 & 1577 \\
\hline$\%$ & $13 \%$ & $16 \%$ \\
\hline Nombre de segments & 186 & 214 \\
\hline
\end{tabular}

18 A titre de comparaison le discours de Pauline Green, politicienne de l'émission 1 correspond à 2423 occurrences et représente un peu plus de $21 \%$ de l'ensemble ; celui de Kenneth Baker, autre politicien invité de l'émission 1 correspond à 2761 occurrences, soit $24 \%$ de l'ensemble. On retrouve donc ici quantitativement le fait que les journalistes modérateurs du débat sont plutôt effacés et parlent relativement peu par rapport aux politiciens : leur discours représente entre 13 et $16 \%$ de l'ensemble. On note également que les ordres de grandeur sont cohérents sur les deux émissions.

Un autre élément intéressant concerne le nombre de segments, d'énoncés prononcés. Sur les deux émissions, les journalistes produisent autour de 400 segments alors que les politiciens seulement 255. Pourtant, l'ensemble de la quantité de discours produit est d'au moins un tiers supérieur quantitativement. Cela est essentiellement dû au fait (nous y reviendrons), que les énoncés sont de nature différente: les politiciens argumentent, dans de véritables mouvements discursifs où s'entrelacent des énoncés plus longs. Il est maintenant nécessaire de préciser la nature des énoncés journalistiques produits dans le cadre de l'émission.

\subsection{Méthodologie}

L'enjeu général est assez bien exprimé par Pourtois et Desmet (1997 : 24) :

[...] Comment appréhender scientifiquement la signification subjective? Et comment saisir par un système de connaissances objectives les structures de signification subjectives?

21 La logométrie intégrative que nous prônons permet de prendre en compte un certain nombre d'exigences précédemment énoncées, à savoir le fait d'explorer l'activité langagière dans sa pluridimensionnalité, ainsi que celui d'objectiver une lecture des corpus.

Un «scheme» a donc été mis au point dans l'environnement logiciel «Systemic Coder» devenu maintenant UAM Corpus Tools ${ }^{3}$. Il s'agit d'une grille multidimensionnelle permettant d'étiqueter des segment (énoncés) en leur attribuant des caractéristiques. A la fin du processus, on aboutit à une base de données, interrogeable à partir de chaque critère (autour de 140 retenus), que l'on peut également combiner avec les opérateurs booléens. Le processus d'identification des segments ne va pas de soi et nous avons hésité entre plusieurs unités a priori (unités syntaxiques, propositions, groupes de souffle etc.) pour finalement considérer que l'unité 
opérationnelle dans notre étude était spécifique et se déterminait par la combinaison des critères qui lui était attribuée. Ainsi, si au cours de l'opération manuelle d'étiquetage des unités discursives, un critère devait apparaître ou disparaître, cela était le signe qu'un bornage était nécessaire, séparant ainsi deux segments de nature différente, qui ne combinaient pas les mêmes critères. Le squelette de cette grille est constitué par le modèle à 5 places développé en linguistique interactionnelle par R. Vion (1995), le niveau séquentiel est considéré (Adam : 1992), ainsi que les modes énonciatifs tels que l'effacement, l'opposition, etc ${ }^{4}$. Ces différents concepts ont été présentés in extenso lors d'études préalables, ils seront rappelés au fil de l'analyse.

Du point de vue statistique, l'analyse quantitative faite par le logiciel comptabilise dans un premier temps en valeur absolue les segments tels qu'ils ont été bornés au fil de l'analyse. Lorsque deux échantillons indépendants sont comparés, comme celui constitué par les énoncés de Sissons d'une part et celui de Dimbleby d'autre part, le logiciel permet la mise en place de $t$-tests ou t-stats. Ce traitement statistique a pour avantage de corriger les variations individuelles extrêmes dans un échantillon de petite taille en considérant comme corpus de référence la population statistique dans son ensemble, suivant Oakes (1998:11-12) :

The $t$-test tests the difference between two groups for normally-distributed interval data where the mean and standard deviation are appropriate measures of central tendency and variability of the scores. We use a t-test rather than a z-score whenever we are dealing with small samples (where either group has less than 30 items). [...]

In two sample studies, the means from two different groups (often an experimental group and a control group) are compared to determine whether the means of these two groups differ for reasons other than pure chance.

The normal distribution is made up of individual scores. The sampling distribution of means, is a distribution which, instead of being made of individual scores, is composed of class means, and also describes a symmetric curve. The means of all groups within a population are much more similar to each other than the individual scores in a single group are to the group mean, because the mean smooths out the effect of extreme individual scores.

Même si les échantillons sont de petite taille, la prédiction est possible et c'est tout l'intérêt de ce traitement statistique. Ainsi, dans les tableaux qui vont suivre, la colonne $t$-stat montre une valeur et un nombre de croix $(+,++,+++)$, comme ici pour les genres discursifs :

Tableau $n^{\circ} 2$. Exemple de traitement des $t$-stats

\begin{tabular}{|c|c|c|c|c|c|c|c|c|}
\hline \multirow[b]{2}{*}{ Feature } & \multicolumn{4}{|l|}{ peter1 } & \multicolumn{4}{|l|}{ david2 } \\
\hline & Mean & $\mathbf{N}$ & TStat & & Mean & $\mathbf{N}$ & TStat & \\
\hline PLACE-MODULAIRE & \multicolumn{4}{|l|}{186} & \multicolumn{4}{|l|}{214} \\
\hline GENRE & \multicolumn{4}{|l|}{186} & \multicolumn{4}{|l|}{214} \\
\hline débat & $61.8 \%$ & 115 & 3.71 & +++ & $78.5 \%$ & 168 & 3.71 & +++ \\
\hline plaisanterie & $9.1 \%$ & 17 & 3.29 & +++ & $1.9 \%$ & 4 & 3.29 & +++ \\
\hline
\end{tabular}




\begin{tabular}{|l|l|l|l|l|l|l|l|l|}
\hline présentation & $16.7 \%$ & 31 & 6.22 & +++ & $0.5 \%$ & 1 & 6.22 & +++ \\
\hline interview & $8.6 \%$ & 16 & 1.95 & + & $15.0 \%$ & 32 & 1.95 & + \\
\hline
\end{tabular}

La valeur $t$-stat dans la $3^{\text {ème }}$ colonne est d'autant plus élevée que l'écart est important et significatif entre les deux échantillons considérés. Au-delà du nombre d'items comparés en valeur absolue, l'écart est bien le facteur déterminant. On voit par exemple ici que pour les segments relevant de la "présentation ", la valeur est de 6.22 pour seulement 1 énoncé de David Dimbleby, contre 31 pour Sissons. Les croix indiquent quelle est la marge d'erreur, ainsi, un + correspond à $10 \%, 2++: 5 \%$ et avec +++ la marge d'erreur est réduite à $2 \%$. Cela signifie entre autres que l'écart est stabilisé. Quel que soit le nombre éventuel d'énoncés supplémentaires, l'écart reste le même et donc la différence de stratégie est statistiquement (objectivement) significative.

\subsection{Les stratégies quantifiées}

Nous l'avons précisé en introduction, la logométrie est ontologiquement comparative. Ainsi, le discours des journalistes modérateurs ne peut s'identifier qu'en le confrontant à celui des autres participants. Dans les paragraphes suivants, nous allons tenter de déterminer les critères saillants par rapport aux politiciens, au public participant et aux experts invités.

\subsubsection{Les genres et types de discours, le niveau séquentiel}

Pour ce qui est des sous-genres constitutifs du débat télévisé, nous avons retenu 5 modules possibles, les phases de débat, de polémique, de plaisanterie, la conversation anodine, la présentation ou bien l'interview. La présence de la plaisanterie peut surprendre, mais il s'agit là d'un élément culturel définitoire pour Question Time. En effet, le débat n'est pas uniquement une mise en interaction de politiciens, hommes et femmes de pouvoirs, mais il appartient également au divertissement télévisé. Les journalistes entre autres sont souvent auteurs de commentaires humoristiques, voici ce que dit Sissons à propos de Kenneth Baker dans l'ouverture de la première émission :

(1) During his ministerial career/ he introduced so many important and controversial reforms/ that it's said the worst job to have in politics/ is the one after Kenneth Baker//

Le ton est apparemment donné, l'émission est détendue, l'invité concerné est montré à l'écran en train de rire après l'énoncé du journaliste.

Le tableau ci-dessous montre que les journalistes sont moins au centre des débats que les autres participants : c'est normal, ils le modèrent a priori. De même, ils sont les interviewers au besoin, et les seuls à pouvoir endosser ce rôle. Ils assurent la présentation de certains faits, éléments contextuels, qu'ils rappellent au téléspectateur pour plus de clarté. 
Tableau $n^{\circ}$ 3. Les genres de discours

\begin{tabular}{|c|c|c|c|c|c|c|c|c|c|c|c|c|c|c|c|c|}
\hline \multirow[b]{2}{*}{ Feature } & \multicolumn{4}{|c|}{ Expert } & \multicolumn{3}{|c|}{ Public } & \multicolumn{5}{|c|}{ politiciens } & \multicolumn{4}{|c|}{ journalistes } \\
\hline & Mean & $\mathbf{N}$ & IStat & & Mean & $\mathbf{N}$ & IStat & & Mean & $\mathbf{N}$ & IStat & & Mean & $\mathrm{N}$ & TStat & \\
\hline $\begin{array}{l}\text { PLACE- } \\
\text { MODULAIRE }\end{array}$ & \multicolumn{4}{|c|}{54} & \multicolumn{3}{|c|}{44} & \multicolumn{5}{|c|}{255} & \multicolumn{4}{|c|}{401} \\
\hline GENRE & \multicolumn{4}{|c|}{54} & \multicolumn{3}{|c|}{44} & \multicolumn{5}{|c|}{255} & \multicolumn{4}{|c|}{401} \\
\hline débat & $92.6 \%$ & 50 & 2.47 & $+1+$ & $100.0 \%$ & 44 & 3.49 & $+1+$ & $87.1 \%$ & 222 & 3.67 & $+1+$ & $70.8 \%$ & 284 & 6.52 & +++ \\
\hline polémique & $7.4 \%$ & 4 & 0.94 & & $0.0 \%$ & 0 & 1.53 & & $8.2 \%$ & 21 & 3.20 & $+H$ & $2.7 \%$ & 11 & 2.80 & ++ \\
\hline plaisanterie & $0.0 \%$ & 0 & 1.29 & & $0.0 \%$ & 0 & 1.16 & & $0.0 \%$ & 0 & 3.34 & $+H$ & $5.2 \%$ & 21 & 4.41 & ++ \\
\hline $\begin{array}{l}\text { conversation- } \\
\text { anodine }\end{array}$ & $0.0 \%$ & 0 & 1.16 & & $0.0 \%$ & 0 & 1.04 & & $4.7 \%$ & 12 & 3.26 & $+1+$ & $1.2 \%$ & 5 & 1.99 & + \\
\hline présentation & $0.0 \%$ & 0 & 1.61 & & $0.0 \%$ & 0 & 1.44 & & $0.0 \%$ & 0 & 4.17 & +++ & $8.0 \%$ & 32 & 5.53 & +++ \\
\hline interview & $0.0 \%$ & 0 & 1.99 & + & $0.0 \%$ & 0 & 1.78 & + & $0.0 \%$ & 0 & 5.20 & $+1+$ & $12.0 \%$ & 48 & 6.92 & $+1+$ \\
\hline
\end{tabular}

31 Le niveau séquentiel nous permet de descendre plus précisément dans ce qui fait le cœur de métier du journaliste modérateur de débat. Rappelons que l'unité nommée séquence par Adam (1992: 28) se définit comme une "structure », c'est-à-dire comme :

- un réseau relationnel hiérarchique: grandeur décomposable en parties reliées entre elles et reliées au tout qu'elles constituent ;

- une entité relativement autonome, dotée d'une organisation interne qui lui est propre et donc en relation de dépendance/indépendance avec l'ensemble plus vaste dont elle fait partie.

Cette unité présente l'avantage d'être autonome mais reliée au tout, ce qui permet de déterminer des relations d'interdépendance et donc des stratégies. Adam, considérant plutôt le domaine littéraire a priori en détermine $5:$ narration, description, dialogue, argumentation, explication. Ces types de séquences peuvent s'absorber au fil du discours : dans un débat politique l'activité discursive principale est l'argumentation à laquelle nous pouvons subordonner les quatre autres, pouvant finalement servir ce dessein. Pour l'étude de Question Time, et plus particulièrement en étudiant le discours des journalistes, nous avions ressenti la nécessité d'étendre la catégorisation au concept d'exposition et de mettre en place une catégorie " autre séquence ", destinée à recueillir les énoncés qui n'entraient pas dans les autres catégories a priori. 
Tableau $\mathrm{n}^{\circ} 4$. Les séquences et les types de discours

\begin{tabular}{|c|c|c|c|c|c|c|c|c|c|c|c|c|c|c|c|c|}
\hline \multirow{3}{*}{\begin{tabular}{|l} 
SEQUENCE \\
autre-sequ
\end{tabular}} & \multicolumn{4}{|c|}{ EXPERTS } & \multicolumn{4}{|c|}{ PUBLIC } & \multicolumn{4}{|c|}{ POLITICIENS } & \multicolumn{4}{|c|}{ JOURNALISTES } \\
\hline & \multicolumn{4}{|c|}{54} & \multicolumn{4}{|c|}{44} & \multicolumn{4}{|c|}{255} & \multicolumn{4}{|c|}{401} \\
\hline & $1.9 \%$ & 1 & 4.85 & +++ & $9.1 \%$ & 4 & 3.23 & +++ & $3.5 \%$ & 9 & 12.76 & +++ & $54.4 \%$ & 218 & 17.82 & +++ \\
\hline récit & $0.0 \%$ & 0 & 0.56 & & $0.0 \%$ & 0 & 0.50 & & $1.6 \%$ & 4 & 2.82 & +4 & $0.0 \%$ & 0 & 2.14 & + \\
\hline description & $24.1 \%$ & 13 & 1.35 & & $15.9 \%$ & 7 & 0.26 & & $18.8 \%$ & 48 & 0.75 & & $15.7 \%$ & 63 & 1.28 & \\
\hline argumentation & $61.1 \%$ & 33 & 3.94 & $+1+$ & $65.9 \%$ & 29 & 4.22 & $+1+$ & $63.1 \%$ & 161 & 11.83 & $+1+$ & $13.0 \%$ & 52 & 16.71 & $+1+$ \\
\hline explication & $13.0 \%$ & 7 & 0.69 & & $6.8 \%$ & 3 & 0.77 & & $12.9 \%$ & 33 & 1.77 & + & $8.5 \%$ & 34 & 1.68 & + \\
\hline exposition & $0.0 \%$ & 0 & 1.68 & + & $2.3 \%$ & 1 & 0.77 & & $0.0 \%$ & 0 & 4.38 & +++ & $8.5 \%$ & 34 & 5.43 & +++ \\
\hline $\begin{array}{l}\text { TYPES- } \\
\text { DISCOURS }\end{array}$ & \multicolumn{4}{|c|}{54} & \multicolumn{4}{|c|}{44} & \multicolumn{4}{|c|}{255} & \multicolumn{4}{|c|}{401} \\
\hline des-médias & $3.7 \%$ & 2 & 6.23 & +++ & $4.5 \%$ & 2 & 5.44 & +++ & $3.5 \%$ & 9 & 19.17 & +++ & $78.1 \%$ & 313 & 31.01 & +++ \\
\hline politique & $11.1 \%$ & 6 & 2.48 & $+1+$ & $27.3 \%$ & 12 & 0.33 & & $57.6 \%$ & 147 & 17.33 & $+1+$ & $6.2 \%$ & 25 & 14.43 & $+1+$ \\
\hline $\begin{array}{l}\text { vie- } \\
\text { quotidienne }\end{array}$ & $68.5 \%$ & 37 & 8.56 & $+1+$ & $63.6 \%$ & 28 & 6.73 & +4 & $23.1 \%$ & 59 & 0.03 & & $12.7 \%$ & 51 & 7.53 & H \\
\hline institutionnel & $7.4 \%$ & 4 & 1.49 & & $0.0 \%$ & 0 & 1.34 & & $5.9 \%$ & 15 & 2.26 & + & $2.2 \%$ & 9 & 2.28 & $H$ \\
\hline propagande & $0.0 \%$ & 0 & 1.41 & & $4.5 \%$ & 2 & 0.47 & & $9.0 \%$ & 23 & 6.41 & $H$ & $0.0 \%$ & 0 & 5.52 & $H+$ \\
\hline économique & $0.0 \%$ & 0 & 0.48 & & $0.0 \%$ & 0 & 0.43 & & $0.8 \%$ & 2 & 1.20 & & $0.2 \%$ & 1 & 0.69 & \\
\hline technocratique & $9.3 \%$ & 5 & 6.82 & +4 & $0.0 \%$ & 0 & 0.66 & & $0.0 \%$ & 0 & 1.90 & + & $0.5 \%$ & 2 & 1.31 & \\
\hline
\end{tabular}

Les énoncés étiquetés description ou exposition renvoient à l'ouverture de la première émission faite par Sissons. Ce dernier se livre à une introduction sur le mode humoristique pour présenter ses invités en lisant un texte préparé en amont, en voici quelques exemples :

Description :

(2) and now backbencher for the man who led the Heath team in the leadership fight against Margaret Thatcher /

(3) Pauline Green is the Labour member of the European parliament for London North/

Exposition :

(4) there was the irony of him in terms of being the last person on the bridge of SS Thatcher as it slipped beneath the waves

(5) that it's said the worst job to have in politics is the one after Kenneth Baker

Ces énoncés correspondent assez bien au mode de discours que C. Smith (2003) nomme « report » et que nous ne connaissions pas au moment de la rédaction de la thèse.

Le type de discours nommé "autres séquences » est marqué comme saillant par rapport aux autres participants. Cette catégorie créée pour les journalistes correspond aux segments que nous n'arrivions pas à étiqueter suivant les types de séquences proposés par Adam, à savoir le récit, la description, l'argumentation, l'explication. La nécessité de trouver d'autres types séquentiels pour analyser la production des modérateurs de débats démontre sa spécificité. Cette " autre séquence » agrège 4 types d'énoncés aux fonctions très particulières correspondant au rôle du meneur de jeu interactionnel, de chef d'orchestre du débat :

Autorisation : donner la parole.

(6) Gentleman at the back

Questionnement (mode de l'interview) :

(7) Can you explain « brutalize " ?

Évaluation (ponctuation de la fin d'une intervention)

(8) OK, Thank you

Reformulations : 
(9) M. Portillo there have been a number of points made: the unemployment figures are phoney, people are suffering pains despite signs of recovery.

$$
\begin{aligned}
& \text { (10) which comes from Brian Davis... } \\
& \text { (11) and Barry Panto / a solicitor / will ask it } \\
& \text { (12) / who's a manufacturer's agent / Sir... } \\
& \text { (13) who's a research assistant / Mr Davis ... } \\
& \text { (14) who's a chartered accountancy student / Sir... }
\end{aligned}
$$

ême, le type de discours " des médias » renvoie à des réalités du même ordre : les énoncés consistent à présenter / introduire une question ou un participant :

La structure est toujours la même, soit il s'agit d'un GN en apposition (11) dont on peut considérer qu'il constitue une relative réduite avec effacement de l'élément "Whiz », ou bien d'une relative appositive (10), (12), (13), (14). La présence d'une telle structure est intéressante ici: Dubois-Charlier et Vautherin (1997: 82) notent que la relative appositive est rare à l'oral, essentiellement à cause de l'organisation complexe de l'information qu'elle induit. Ceci est statistiquement contestable ici : nous trouvons pléthore de ces énoncés pour les journalistes. Leur production dépend sans doute de ce que "l'on a à faire » : quoi de mieux en effet qu'une relative appositive pour donner une information secondaire à propos du profil d'un intervenant? On doit donc considérer qu'il s'agit d'une marque du genre liée aux débats mais également une marque du rôle, car cette structure est spécifique du discours des journalistes : aucun autre participant ne l'utilise (avec who en introducteur). En revanche, les journalistes n'introduisent que très rarement des relatives appositives avec le pronom relatif which, ce qui peut arriver plus fréquemment pour les autres invités.

\begin{tabular}{|c|c|c|c|c|c|c|c|c|c|c|c|c|c|c|c|c|}
\hline & \multicolumn{4}{|c|}{ Experts } & \multicolumn{4}{|c|}{ Public } & \multicolumn{4}{|c|}{ Politiciens } & \multicolumn{4}{|c|}{ Journalistes } \\
\hline $\begin{array}{l}\text { PLACE-SUBJECTIVE- } \\
\text { INTERNE }\end{array}$ & \multicolumn{4}{|c|}{54} & \multicolumn{4}{|c|}{44} & \multicolumn{4}{|c|}{255} & \multicolumn{4}{|c|}{401} \\
\hline APPELLATIFS & \multicolumn{4}{|c|}{54} & \multicolumn{4}{|c|}{44} & \multicolumn{4}{|c|}{255} & \multicolumn{4}{|c|}{401} \\
\hline appellatifs-type-inteme & $1.9 \%$ & 1 & 4.34 & $+1+$ & $2.3 \%$ & 1 & 3.82 & $+1+$ & $2.7 \%$ & 7 & 11.55 & $+1+$ & $48.1 \%$ & 193 & 16.41 & +++ \\
\hline $\begin{array}{l}\text { APELLATIFS- } \\
\text { SPECIFIQUES }\end{array}$ & \multicolumn{4}{|c|}{54} & \multicolumn{4}{|c|}{44} & \multicolumn{4}{|c|}{255} & \multicolumn{4}{|c|}{401} \\
\hline nom-complet & $1.9 \%$ & 1 & 1.75 & + & $2.3 \%$ & 1 & 1.46 & & $0.4 \%$ & 1 & 5.65 & $+1+$ & $14.5 \%$ & 58 & 7.05 & +++ \\
\hline titre & $0.0 \%$ & 0 & 0.39 & & $0.0 \%$ & 0 & 0.35 & & $0.0 \%$ & 0 & 1.01 & & $0.5 \%$ & 2 & 1.33 & \\
\hline prênom & $0.0 \%$ & 0 & 0.39 & & $0.0 \%$ & 0 & 0.35 & & $0.0 \%$ & 0 & 1.01 & & $0.5 \%$ & 2 & 1.33 & \\
\hline nom-complet-et-titre & $0.0 \%$ & 0 & 0.28 & & $0.0 \%$ & 0 & 0.25 & & $0.0 \%$ & 0 & 0.71 & & $0.2 \%$ & 1 & 0.94 & \\
\hline no-specifique-interne & $0.0 \%$ & 0 & 3.60 & +++ & $0.0 \%$ & 0 & 3.22 & +++ & $2.4 \%$ & 6 & 8.36 & +++ & $32.4 \%$ & 130 & 11.92 & +++ \\
\hline $\begin{array}{l}\text { APPELLATIFS- } \\
\text { GENERIQUES }\end{array}$ & \multicolumn{4}{|c|}{54} & \multicolumn{4}{|c|}{44} & \multicolumn{4}{|c|}{255} & \multicolumn{4}{|c|}{401} \\
\hline pronoms & $1.9 \%$ & 1 & 2.61 & +1 & $0.0 \%$ & 0 & 2.71 & $+1+$ & $2.4 \%$ & 6 & 6.59 & +1 & $23.7 \%$ & 95 & 9.16 & +++ \\
\hline autre-gn & $0.0 \%$ & 0 & 1.95 & + & $2.3 \%$ & 1 & 1.09 & & $0.0 \%$ & 0 & 5.08 & $+1+$ & $11.2 \%$ & 45 & 6.42 & +++ \\
\hline no-generique-interne & $0.0 \%$ & 0 & 2.12 & + & $0.0 \%$ & 0 & 1.90 & + & $0.4 \%$ & 1 & 5.24 & $+1+$ & $13.2 \%$ & 53 & 7.09 & +++ \\
\hline
\end{tabular}

Comme le montre le tableau suivant, les appellatifs sont également abondamment utilisés par les journalistes, pour servir les mêmes fonctions :

Tableau $n^{\circ} 5$. Les appellatifs

39 Les modérateurs font par nature circuler la parole. Ils nomment ou identifient donc les participants pour leur donner la parole; nous avons déjà vu quelques exemples plus haut : gentleman at the back, Mr Davis, M. Portillo... etc. 


\subsubsection{Les mises en scènes énonciatives}

Le concept a été forgé par Vion (1998) :

La notion de mise en scène concerne la façon dont les sujets construisent les opinions qu'ils mettent en circulation. Il s'agit, notamment, de savoir quelles sont les sources énonciatives construites dans les messages et de répondre à des questions comme: 'Le locuteur donne-t-il l'impression de parler seul ?', 'Fait-il parler d'autres personnes?', 'Ces autres voix sont-elles identifiables ou non?', 'Comment ces diverses voix s'articulent-elles dans la parole du locuteur?' et 'Comment celui-ci se positionne-t-il vis-à-vis des opinions ainsi construites ?'

Cinq types de mise en scène ont été formalisés, intégrant chacun des sous-type correspondant à des postures énonciatives spécifiques relevant parfois d'un marquage explicite ou identifiable :

- l'unicité (le locuteur parle seul), les énoncés intègrent des marques de subjectivité : Unicité explicite : présence de marqueurs tels que I think, my opinion is, my view is. Unicité implicite : absence de marqueurs introducteurs d'opinions, mais l'énoncé relève d'une prise d'opinion.

- la dualité (deux énonciations sont construites et se chevauchent) :

Actes indirects et paradoxaux (hérités de Searle) : promettre et en même temps menacer Humour : décalage énonciatif, ironie flagrante pour amuser

Attitude modale : double énonciation avec marqueur modal (modus / dictum) Glose méta-énonciative : énoncé en commentant un autre, surplomb autonymique - le parallélisme (le locuteur parle avec quelqu'un ou un groupe de personnes), Parallélisme diaphonique: le locuteur s'appuie sur un autre en présence (as you say) Parallélisme exophonique : le locuteur s'appuie sur une source identifiée mais absente (citation : according to ...)

Parallélisme polyphonique : le locuteur va dans le même sens qu'un groupe non identifié clairement : as we all know ...

Porte-parole : le locuteur se présente comme le porte-parole d'un groupe plus ou moins identifié (We ...)

- l'opposition (le locuteur parle contre quelqu'un), les énoncés intègrent un marqueur de négation, un marqueur d'opposition.

Opposition diaphonique : opposition marquée contre un participant à l'échange Opposition exophonique : opposition marquée contre un « absent » (groupe ou personne) mais identifié : You IRA terrorists.

Opposition polyphonique : opposition marquée contre un groupe non identifiable - l'effacement (le locuteur semble absent de son discours, les marques subjectives sont gommées).

Enonciation abstraite : les règlements et textes de loi

Enonciation historique (héritée de Benvéniste) : présentation factuelle « qui parle d'ellemême ».

Quels positionnements énonciatifs nos journalistes modérateurs vont-ils donc réaliser au regard des outils présentés ci-dessus? 
Tableau $n^{\circ} 6$. Les mises en scènes énonciatives

\begin{tabular}{|c|c|c|c|c|c|c|c|c|c|c|c|c|c|c|c|}
\hline & \multicolumn{4}{|c|}{ Experts } & \multicolumn{3}{|c|}{ Public } & \multicolumn{4}{|c|}{ Politiciens } & \multicolumn{4}{|c|}{ Journalistes } \\
\hline $\begin{array}{l}\text { PLACE- } \\
\text { ENONCLATIVE }\end{array}$ & & 54 & & & & 44 & & & & 55 & & & 401 & & \\
\hline e-en-scène & $98.1 \%$ & $532^{2}$ & & m & $97.7 \%$ & \begin{tabular}{|l|l|}
43 & 2.09 \\
\end{tabular} & + & $97.3 \%$ & 248 & 5.84 & $m$ & $79.1 \%$ & $\begin{array}{r}517 \\
317\end{array}$ & $7.97+$ & + \\
\hline $\begin{array}{l}\text { tabilite- } \\
\text { nciative }\end{array}$ & $0.0 \%$ & & & & $2.3 \%$ & $\begin{array}{lll}1 & 0.81\end{array}$ & & $0.8 \%$ & & & & & 5 & 0.53 & \\
\hline fragment & $1.9 \%$ & 12 & & 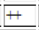 & $0.0 \%$ & 02.44 & $\ldots$ & $2.0 \%$ & 5 & 5.90 & $\mp$ & $19.7 \%$ & 798 & $8.12+$ & 世 \\
\hline UNCIIE & & 54 & & & & 44 & & & 25 & 55 & & & 401 & & \\
\hline $\begin{array}{l}\text { cité-non- } \\
\text { cruese }\end{array}$ & $42.6 \%$ & & & + & $29.5 \%$ & \begin{tabular}{l|l}
13 & 5.16
\end{tabular} & ++ & $62.4 \%$ & 159 & 1.08 & & & $\begin{array}{l}\% 295 \\
0\end{array}$ & $5.36+$ & + \\
\hline Explicite & $11.1 \%$ & 60 & & & $29.5 \%$ & $\begin{array}{ll}13 & 5.56 \\
\end{array}$ & 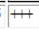 & $12.9 \%$ & $=\longdiv { 3 3 }$ & 3.64 & $F$ & $2.0 \%$ & 8 & $6.62+$ & m \\
\hline mplicite & $44.4 \%$ & 246. & & $+m$ & $38.6 \%$ & \begin{tabular}{|l|l|}
17 & 4.67 \\
\end{tabular} & + & $22.0 \%$ & 56 & 4.05 & m & $3.5 \%$ & 149 & $9.84+$ & +m \\
\hline DUALITE & 54 & & & & 44 & & & 255 & & & & 401 & & & \\
\hline quée & $83.3 \%$ & \begin{tabular}{l|l}
45 & 3. \\
.
\end{tabular} & & + & $93.2 \%$ & $\begin{array}{lll}41 & 4.83\end{array}$ & + & $80.0 \%$ & 204 & 8.84 & +m & $38.4 \%$ & $\begin{array}{lll}6 & 154 & 1\end{array}$ & $1358+$ & +H \\
\hline actes-indirects & $0.0 \%$ & $\begin{array}{ll}0 & 0 . \\
\end{array}$ & & & $0.0 \%$ & \begin{tabular}{l|l}
0 & 0.79
\end{tabular} & & $2.0 \%$ & 5 & 1.09 & & $1.2 \%$ & 5 & 0.20 & \\
\hline $\begin{array}{l}\text { ylose-méta- } \\
\text { inonciative }\end{array}$ & $3.7 \%$ & $\begin{array}{ll}2 & 2 . \\
\end{array}$ & & + & $0.0 \%$ & $\begin{array}{l}0 \\
2\end{array} 2.84$ & + & $1.2 \%$ & 3 & 7.74 & + & $26.2 \%$ & 1051 & $10.25+$ & ++ \\
\hline attitude-modale & $11.1 \%$ & $6 \frac{0}{6}$ & & & $4.5 \%$ & \begin{tabular}{|l|l}
2 & 1.12 \\
\end{tabular} & & $14.1 \%$ & $=\overline{36}$ & 3.29 & $=$ & $6.5 \%$ & $=\frac{126}{22}$ & $2.84+$ & m \\
\hline humour & $0.0 \%$ & $\begin{array}{lll}0 & 1 .\end{array}$ & & & $0.0 \%$ & \begin{tabular}{l|l}
0 & 1.29
\end{tabular} & & $0.0 \%$ & 0 & 3.74 & $+m$ & $6.5 \%$ & $\begin{array}{ll}26 & 4 \\
\end{array}$ & $4.94+$ & +m \\
\hline PARALLELISMIE & & 54 & & & & 44 & & & 25 & 55 & & & 401 & & \\
\hline $\begin{array}{l}\text { paralalelisme-non- } \\
\text { marque }\end{array}$ & $68.5 \%$ & 371. & & + & $59.1 \%$ & $\begin{array}{ll}26 & 0.39\end{array}$ & & $64.7 \%$ & 165 & 3.37 & + & $48.9 \%$ & $\begin{array}{lll}6 & 196 & 4\end{array}$ & $4.39+$ & + \\
\hline p-diaphonique & $0.0 \%$ & 0.1. & & + & $6.8 \%$ & 30.20 & & $2.4 \%$ & 6 & 3.09 & $\ldots$ & $9.2 \%$ & 373 & $3.86+$ & $\Pi$ \\
\hline -essephopigure & $0.0 \%$ & $\frac{1}{1}$ & & + & $4.5 \%$ & \begin{tabular}{|l|l|}
2 & 0.03 \\
\end{tabular} & & $5.1 \%$ & 113 & 0.43 & & $5.0 \%$ & $=\frac{20}{20}$ & $\mid 0.48$ & \\
\hline p-polyphonique & $14.8 \%$ & \begin{tabular}{l|l}
8 & 1.
\end{tabular} & & + & $25.0 \%$ & 114.28 & $\ldots$ & $12.2 \%$ & 31 & 2.94 & $m$ & $2.7 \%$ & 115 & $5.86+$ & + \\
\hline te-pa & $14.8 \%$ & 80. & & & $2.3 \%$ & \begin{tabular}{|l|l|}
1 & 2.13 \\
\end{tabular} & + & $12.9 \%$ & 33 & 0.20 & & $13.2 \%$ & 530 & 0.54 & \\
\hline OPPOSTIION & & 54 & & & & 44 & & & 25 & 55 & & & 401 & & \\
\hline $\begin{array}{l}\text { opposition-non- } \\
\text { marquée }\end{array}$ & $55.6 \%$ & 300. & & & $72.7 \%$ & \begin{tabular}{l|l}
32 & 1.96
\end{tabular} & + & $67.8 \%$ & 173 & 3.70 & + & $51.6 \%$ & 6 2074 & $4.20+$ & + \\
\hline o-diaphonique & $1.9 \%$ & 12 & & 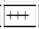 & $2.3 \%$ & 112.09 & + & $4.7 \%$ & 12 & 4.61 & \# & $19.7 \%$ & 796. & $6.74+$ & 世 \\
\hline 1-8xpophoniguse & $1.9 \%$ & 10 & & & $0.0 \%$ & $\begin{array}{ll}0 & 1.42 \\
\end{array}$ & & $9.0 \%$ & 23 & 4.92 & 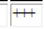 & $1.7 \%$ & $=\frac{3}{7}$ & $3.51+$ & m \\
\hline o-polyphonique & $37.0 \%$ & 206. & & 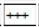 & $22.7 \%$ & 102.28 & + & $15.7 \%$ & 40 & 2.28 & $\#$ & $5.0 \%$ & 206 & $6.43+$ & 册 \\
\hline o-ironique & $1.9 \%$ & & & & $0.0 \%$ & $\begin{array}{ll}0 & 0.56 \\
\end{array}$ & & $0.0 \%$ & $=\overline{0}$ & 1.60 & & $1.0 \%$ & $=\frac{1}{4}$ & 1.21 & \\
\hline FACEMENT & & 54 & & & & 44 & & & 25 & 55 & & & 401 & & \\
\hline $\begin{array}{l}\begin{array}{l}\text { effacement-non- } \\
\text { marquué }\end{array} \\
\end{array}$ & $94.4 \%$ & $\begin{array}{ll}51 & 3 .\end{array}$ & & + & $81.8 \%$ & $\begin{array}{ll}36 & 1.33\end{array}$ & & $81.2 \%$ & & 3.56 & + & $64.3 \%$ & $1 \% 2585$ & 5.99 & \\
\hline $\begin{array}{l}\text { Enonciation- } \\
\text { abstraite }\end{array}$ & $0.0 \%$ & $\begin{array}{lll}0 & 0 .\end{array}$ & & & $0.0 \%$ & $\begin{array}{lll}0 & 0.43\end{array}$ & & $1.2 \%$ & 3 & 2.43 & m & $0.0 \%$ & 0 & $1.85+$ & \\
\hline $\begin{array}{l}\text { nonciation- } \\
\text { historique }\end{array}$ & $3.7 \%$ & $\begin{array}{ll}2 & 2 .\end{array}$ & & + & $15.9 \%$ & $\begin{array}{lll}7 & 0.36\end{array}$ & & $14.9 \%$ & & 0.48 & & $14.7 \%$ & $\begin{array}{ll}059 & 0 .\end{array}$ & 0.55 & \\
\hline
\end{tabular}

Le tableau global donne un certain nombre d'indications intéressantes. Le nombre de fragments est plus important que pour les autres participants: beaucoup de $O K$, ou simplement des No, prononcés par les journalistes ponctuent le débat. Nous avons donc identifié comme fragments les monosyllabes qui renvoient à la fonction d'évaluation ou bien de régulation de la parole. Ces énoncés correspondent à la catégorie de l'autre séquence, identifiée plus haut ; ils peuvent relever de plusieurs types de mises en scène, essentiellement dans les modes de l'opposition (No!), ou du parallélisme (OK).

Deux modes se font écho et reprennent un certain nombre d'observations déjà formulées. L'unicité est non marquée, ce qui signifie que le discours n'est pas introduit par des marqueurs de subjectivité explicites tels que "I think that ", ce qui est attendu (espéré ?) pour un journaliste. De même, on voit que l'effacement non marqué est le plus faible, ce qui implique a contrario que les journalistes sont bien effacés ( $64 \%$ contre 94 , 82 et $81 \%$ ). On note que l'énonciation historique est à presque $15 \%$, le discours des modérateurs se présente comme objectivé, gommant les marques de la subjectivité.

Les modes de la dualité et de l'opposition retiennent notre attention. Ils permettent clairement de distinguer les journalistes des autres participants. Voici quelques exemples :

Dualité : glose méta-énonciative

(15) We've had a lot of questions ...

(16) Let's leave that question for the moment

(17) Thank you ...

(18) You must stop!

Les quatre exemples précédents sont des gloses discursives sur la conduite des débats ou en surplomb du débat. Le terme méta-linguistique question apparaît déjà deux fois. 
On pourrait parler d'énoncés « méta-interactionnels » dont la fonction est de cadrer le débat.

Le mode de l'opposition est significatif car il met en lumière un phénomène de chiasme. Les journalistes, fidèles à eux-mêmes, sont dans le diaphonique, le duel en " one-to-one " alors que tous les autres participants s'opposent sur le mode polyphonique à des personnes ou groupes qui ne sont pas identifiées clairement. Nous avions préalablement mis en avant (Rouveyrol et Bendinelli, 2011) que le discours du politicien consistait essentiellement à parler « avec certains » (ses sympathisants, les membres du parti etc.) et contre d'autres (tous les opposants possibles). Voici quelques exemples d'opposition diaphonique pour les journalistes (outre l'exemple 18 vu plus haut) :

(19) Where have you gone wrong?

(20) That wasn't very chivalrous!

L'opposition est utilisée ici comme un mode énonciatif permettant de stimuler la parole, de susciter une réaction ou un commentaire, voire de clôturer une intervention (18). A ce propos nous avons déterminé récemment (Rouveyrol, 2018) que bien qu'effacés, les journalistes meneurs du jeu interactionnel pouvaient produire des "taxèmes", ces énoncés qui placent leur énonciateur au-dessus des autres (Kerbrat-Orecchioni, 1992 : 95 et seq.). Ainsi, pour clôturer un module thématique et passer à la question suivante, le journaliste peut être amené, avec plus ou moins de succès, à renier aux participants le droit de s'exprimer. On parlera alors de «non-accommodation » : le compromis n'est plus possible, la phase modulaire est définitivement close.

Plus globalement, l'analyse des mises en scène corrobore l'idée que les journalistes sont au cœur du débat, sans pour autant débattre eux-mêmes : ils ne donnent pas leur avis (le mode de l'unicité est faiblement représenté). L'utilisation de monosyllabes (fragments) montre leur volonté d'être impliqués profondément dans le jeu interactionnel qu'ils tentent de réguler avec le plus de réactivité possible. Cette dimension méta-interactionnelle se retrouve également dans le fait que le «discours sur le discours » est important, voire définitoire : le nombre de gloses méta-énonciatives est 10 fois supérieur pour les journalistes que pour les autres types de participants.

\subsection{Le discours des journalistes : vers une possible synthèse}

50 Un certain nombre de dimensions peuvent désormais être mises en perspective pour caractériser le discours des journalistes modérateurs de débats télévisés :

- aspect diaphonique :

51 Ils gèrent le débat sur le plateau et leur discours est toujours en relation avec un autre participant, contrairement aux politiciens plus «polyphoniques». Les gloses métaénonciatives sont nombreuses, de même que les interrogatives directes. Ils conduisent, stimulent, cadrent, font progresser les débats.

- aspect fragmenté

Les énoncés des journalistes sont souvent des fragments cours disséminés au fil de l'interaction. Un exemple assez parlant serait le suivant :

(22) OK / I / No / I think / We must move on Shirley

Pas moins de 5 mises en scène énonciatives sont ici juxtaposées.

- aspect effacé 

énoncés des journalistes et d'autre part d'un corpus de référence constitué dans ce cas par l'ensemble des interactions 1 et 2, dans lesquelles agissent les journalistes, est capable de proposer une liste de mots clefs significative pour le discours des journalistes. Le tableau obtenu est le suivant :

Tableau $n^{\circ}$ 7. Les mots-clefs des journalistes

\begin{tabular}{|c|c|c|c|c|}
\hline \multicolumn{2}{|c|}{ Keyword Types: } & \multirow{2}{*}{$\begin{array}{l}7 \\
\text { Keyness }\end{array}$} & \multirow{2}{*}{$\begin{array}{l}\text { Keyword Tokens: } 210 \\
\text { Effect }\end{array}$} & \multirow{2}{*}{$\begin{array}{l}\text { Search Hits: } \\
\text { Keyword }\end{array}$} \\
\hline Rank & Freq & & & \\
\hline 1 & 91 & +46.24 & 0.0532 & you \\
\hline 2 & 16 & +23.22 & 0.0103 & thank \\
\hline 3 & 15 & +22.56 & 0.0097 & $\operatorname{man}$ \\
\hline 4 & 14 & +21.94 & 0.009 & row \\
\hline 5 & 42 & +18.01 & 0.0259 & on \\
\hline 6 & 20 & +17.34 & 0.0128 & let \\
\hline 7 & 12 & +16.71 & 0.0077 & gentleman \\
\hline
\end{tabular}

Sept termes sont ainsi identifiés. Le côté diaphonique saute aux yeux avec you. Thank renvoie à la formulation Septdes gloses méta-énonciatives. Man, row, gentleman font partie des GN qui identifient les futurs locuteurs à qui l'on donne la parole au fil du débat: ces termes renvoient à la gestion de l'interaction et plus précisément à la circulation de la parole. On est intéressant. On trouve un certain nombre de hold on, hold on du journaliste qui souhaite rebondir sur une intervention et demander une précision; la préposition existe aussi isolément et renvoie à la thématique traitée : on this, on the matter... Let est souvent corrélé avec la forme réduite de us et concerne également la conduite des débats: let's move on, let me just ask you, let's hear Shirley briefly, etc. On retiendra donc que le marquage lexical ciblé par les mots-clefs pointe vers la dimension métalinguistique, méta-discursive du rôle de modérateur.

\section{Style et variations : Sissons vs Dimbleby}

57 Nous l'avons dit en introduction, nous considérons deux émissions du débat Question Time présentée pour la première par Peter Sissons et pour la seconde par David Dimbleby. Elles datent de 1992 et 1997, le contexte politico-social est différent (nous y reviendrons) mais d'autres caractères les distinguent. 


\subsection{Eléments structurels}

\subsubsection{Les ouvertures}

Les deux journalistes gèrent très différemment les ouvertures des émissions. Sissons se livre à un numéro humoristique pour présenter ses invités en lisant un document écrit. L'ouverture dure 1'30 et représente 16\% de l'ensemble de son discours. Dimbleby se contente du strict minimum : nom des invités, qualité. Son ouverture représente $3 \%$ de l'ensemble de sa production.

\subsubsection{Gestion des tours de paroles}

Cela ne saute pas aux yeux a priori, mais la façon de gérer les phases interactionnelles est différente pour les deux hommes de médias, ce qui impulse une structure différente au programme :

- Sissons : questions + 4 interventions (invités du panel) + public

- Dimbleby : question + un membre du panel + public + un membre du panel + public...

La stratégie de Dimbleby met en place une véritable interaction entre le public et le panel, alors que dans l'émission de Sissons, l'impression suivant laquelle les invités débattent entre eux prédomine.

61 Les téléspectateurs ne semblent pas s'y tromper à en croire les commentaires qu'ils laissent sur le site de la BBC :

I think interaction is what makes it work more. The Peter Sissons [Question Time] style of things is traditional - it's a good formula and it provides good television and good debate, but the debate is debated between the politicians about issues suggested by the audience. (George, viewer)

\subsection{Les systèmes de la discorde}

Le tableau comparatif général donne des informations intéressantes. On note que seulement 8 traits sur 140 sont divergents, ce qui méthodologiquement implique que la grille d'analyse doit être la plus précise mais aussi la plus multidimensionnelle possible. Le fait que peu de traits distinguent les deux journalistes montre que leurs énonciations sont cohérentes : ils font la même chose légèrement différemment.

Une constatation intéressante est que Dimbleby a plus de segments (214) que Sissons (186). Cela ne veut pas dire qu'il parle plus, car en fait il parle moins (1435 occurrences contre 1558 pour Sissons). Cela implique plutôt que ces énoncés sont mieux répartis dans le débat, qu'il est plus présent, ce que nous allons vérifier à plusieurs niveaux.

Tableau ${ }^{\circ}$ 8. Les systèmes divergents (Dimbleby / Sissons)

\begin{tabular}{|c|c|c|c|c|c|c|}
\hline \multirow[b]{2}{*}{ Feature } & \multicolumn{3}{|l|}{ peter1 } & \multicolumn{3}{|c|}{ david2 } \\
\hline & Mean & $\mathbf{N}$ & TStat & Mean & $\mathbf{N}$ & TStat \\
\hline PLACE-MODULAIRE & \multicolumn{3}{|l|}{186} & \multicolumn{3}{|l|}{214} \\
\hline GENRE & \multicolumn{3}{|l|}{186} & \multicolumn{3}{|l|}{214} \\
\hline
\end{tabular}




\begin{tabular}{|c|c|c|c|c|c|c|c|c|}
\hline débat & $61.8 \%$ & 115 & 3.71 & +++ & $78.5 \%$ & 168 & 3.71 & +++ \\
\hline plaisanterie & $9.1 \%$ & 17 & 3.29 & +++ & $1.9 \%$ & 4 & 3.29 & +++ \\
\hline présentation & $16.7 \%$ & 31 & 6.22 & +++ & $0.5 \%$ & 1 & 6.22 & +++ \\
\hline interview & $8.6 \%$ & 16 & 1.95 & + & $15.0 \%$ & 32 & 1.95 & + \\
\hline PLACE-DISCURSIVE & \multicolumn{4}{|l|}{186} & \multicolumn{4}{|l|}{214} \\
\hline SEQUENCE & \multicolumn{4}{|l|}{186} & \multicolumn{4}{|l|}{214} \\
\hline description & $22.6 \%$ & 42 & 3.54 & +++ & $9.8 \%$ & 21 & 3.54 & +++ \\
\hline PLACE-ENONCIATIVE & \multicolumn{4}{|l|}{186} & \multicolumn{4}{|l|}{214} \\
\hline mise-en-scène & $75.8 \%$ & 141 & 1.46 & & $81.8 \%$ & 175 & 1.46 & \\
\hline fragment & $23.1 \%$ & 43 & 1.58 & & $16.8 \%$ & 36 & 1.58 & \\
\hline DUALITÉ & \multicolumn{4}{|l|}{186} & \multicolumn{4}{|l|}{214} \\
\hline glose-méta-énonciative & $15.1 \%$ & 28 & 4.87 & +++ & $36.0 \%$ & 77 & 4.87 & +++ \\
\hline humour & $10.8 \%$ & 20 & 3.25 & +++ & $2.8 \%$ & 6 & 3.25 & +++ \\
\hline PARALLÉLISME & \multicolumn{4}{|l|}{186} & \multicolumn{4}{|l|}{214} \\
\hline p-diaphonique & $5.4 \%$ & 10 & 2.51 & +++ & $12.6 \%$ & 27 & 2.51 & +++ \\
\hline OPPOSITION & \multicolumn{4}{|l|}{186} & \multicolumn{4}{|l|}{214} \\
\hline o-diaphonique & $16.7 \%$ & 31 & 1.44 & & $22.4 \%$ & 48 & 1.44 & \\
\hline EFFACEMENT & \multicolumn{4}{|l|}{186} & \multicolumn{4}{|l|}{214} \\
\hline énonciation-historique & $19.4 \%$ & 36 & 2.43 & +++ & $10.7 \%$ & 23 & 2.43 & +++ \\
\hline
\end{tabular}

\subsubsection{Genres et séquences}

La stratégie différente adoptée pour l'ouverture pèse lourd, elle explique la différence identifiée entre les deux hommes pour les traits d'humour et de description qui sont au moins deux fois plus importants du côté de Sissons.

Au niveau du genre, Dimblely est davantage dans le débat (78 \% contre $62 \%)$, certes son ouverture minimale augmente de fait le nombre d'énoncés au cœur des débats mais ce n'est peut-être pas la seule explication. Le tableau montre que le genre de l'interview est deux fois plus important dans le discours de Dimbleby. Le présentateur de la deuxième émission " cuisine » ses invités pour obtenir ce qu'il veut. C'est là que les hold on, hold on identifiés comme mots-clefs apparaissent. Voici la salve de questions qu'il adresse successivement à Andrew Smith sur le chômage : 
- (23) Andrew Smith what is the/ no further public spending Labour Party going to do? When faced with this with those issues?

- (24) But you've defined the problem, what is Labour going to do about it?

- (25) So they need more money?

\subsubsection{Les mises en scène}

On note que les glose méta-énonciatives $(15 \%$ / 36\%) sont plus nombreuses également pour Dimbleby. Il fait circuler la parole de façon plus intensive et participe activement à la construction du débat.

(26) Are you suggesting...

(27) We must move on Shirley,

(28) Be very brief cos'...

Dans le même esprit, le parallélisme diaphonique est davantage mis en avant (5.4\% / 12,6\%) chez Dimbleby. Il stimule les débats en malmenant parfois ses invités (30), dans un mouvement argumentatif qui s'apparente à la concession: je fais semblant de vous suivre MAIS... .

(29) Shirley Williams, do you want to comment on what he said?

(30) When you said you were using clean figures that nobody could argue with,

(you've excluded that $=$ opposition) ?

\subsubsection{L'opposition}

Ce trait n'est pas identifié comme saillant, pourtant quelque chose d'intéressant est à l'œuvre ici pour tenter de saisir des différences de stratégie, si l'on se penche sur les énoncés. Nous proposons de les organiser sous forme de tableau pour laisser le contraste mieux apparaître.

Tableau n 9. Opposition ciblée Sissons / Dimbleby

\begin{tabular}{|l|l|l|}
\hline $\begin{array}{l}\text { Opposition } \\
\text { contre : }\end{array}$ & Sissons & Dimbleby \\
\hline le public & $\begin{array}{l}\text { (31) We're not talking about specifics at all, } \\
\text { don't don't... } \\
\text { (32) But let's not get too drawn... }\end{array}$ & $\begin{array}{l}\text { (35) Yeah Sir, go on sir... } \\
\text { (36 Yes, anybody else ... }\end{array}$ \\
\hline le panel & $\begin{array}{l}\text { (33) Not even if the Irish did it at the same jamais au public: } \\
\text { time? } \\
\text { (34) Is there really any difference opinion } \\
\text { between you and KB? }\end{array}$ & $\begin{array}{l}\text { (20) Where have you gone wrong? } \\
\text { (37) Did this fudge the figures? } \\
\text { behind in the polls? } \\
\text { (39) Let me pick up on that... }\end{array}$ \\
\hline
\end{tabular}

Les choses sont claires, Sissons se positionne clairement du côté de l'institution, lorsqu'il s'oppose c'est contre le public et bien souvent pour lui demander de se taire (taxème clôturant, (31-32)). L'énoncé 32 sonne d'ailleurs très professoral : vous ne pouvez pas dire cela est le message implicite. En ce qui concerne l'opposition dirigée contre le panel, elle est beaucoup plus douce, le ton est très urbain. 
Dimbleby en revanche ne s'oppose jamais au public, au contraire, il l'exhorte à poursuivre, à parler davantage. Lorsqu'il s'oppose au panel, le ton est bien différent de celui de Sissons, on sent la volonté d'aller au contact (39) voire même de provoquer (20, 37, 38), en posant les questions " qui fâchent ».

71 Une recherche de combinaisons de critères donne corps à cette analyse. La combinaison (argumentation+interview) donne 8 segments pour Dimbleby et 2 pour Sissons ; la combinaison (argumentation+opposition diaphonique) donne 20 segments pour Dimbleby et 15 pour Sissons.

72 Dans l'émission de Dimbleby, la parole n'est jamais vraiment acquise pour les invités qui doivent parfois faire entendre leur voix en amont pour pouvoir parler. Ceci est visible si nous prenons en compte les nombreux « David» qui jalonnent l'interaction : David, can I just, David let me ... En revanche, l'analyste de discours a beau chercher, aucun Peter n'est prononcé dans la première interaction, à croire que lorsque la parole est acquise, il n'y a aucun besoin de la demander.

\subsection{Conclusion provisoire}

$73 \mathrm{Au}$ vu de ce qui vient d'être développé, on peut raisonnablement conclure que Dimbleby se pose en responsable du débat et en participant à part entière ; il bouscule ses invités et privilégie le public. Sissons est quant à lui effacé et semble piloter à distance le débat en donnant la priorité au panel, il est clairement du côté de l'institution. La gestion du débat est traditionnelle, pour ne pas dire « old school ».

La scénographie va tout à fait dans ce sens ; la capture d'écran suivante montre Sissons trônant au centre de la table avec deux invités de chaque côté : ladies on the right, men on the left.

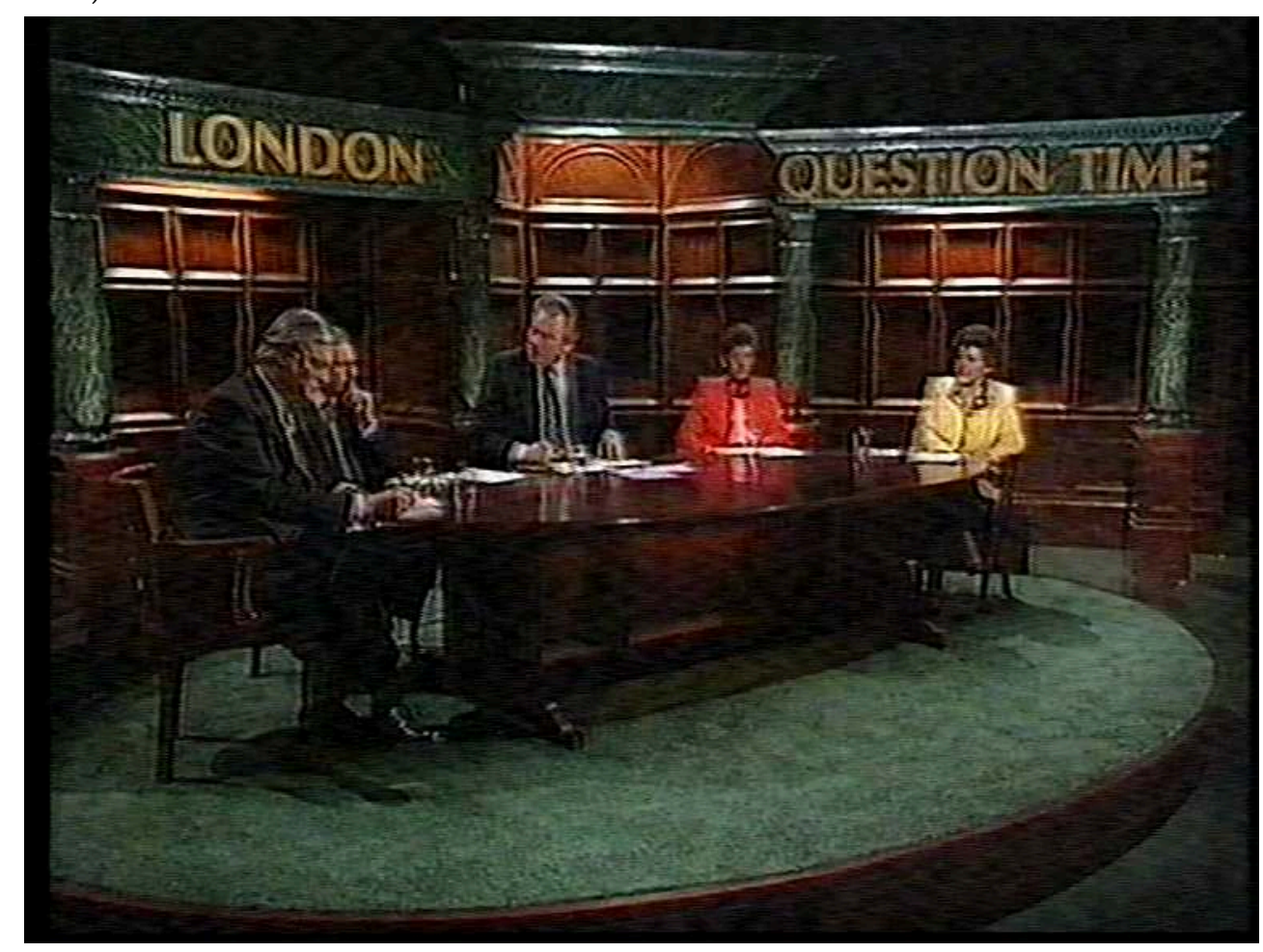


75 Les choses sont différentes pour Dimbleby, dès l'ouverture, il parle face à la caméra avec le public derrière lui. Le message implicite pourrait être : je suis là pour relayer votre parole, cette émission est faite pour vous donner la parole à travers moi.

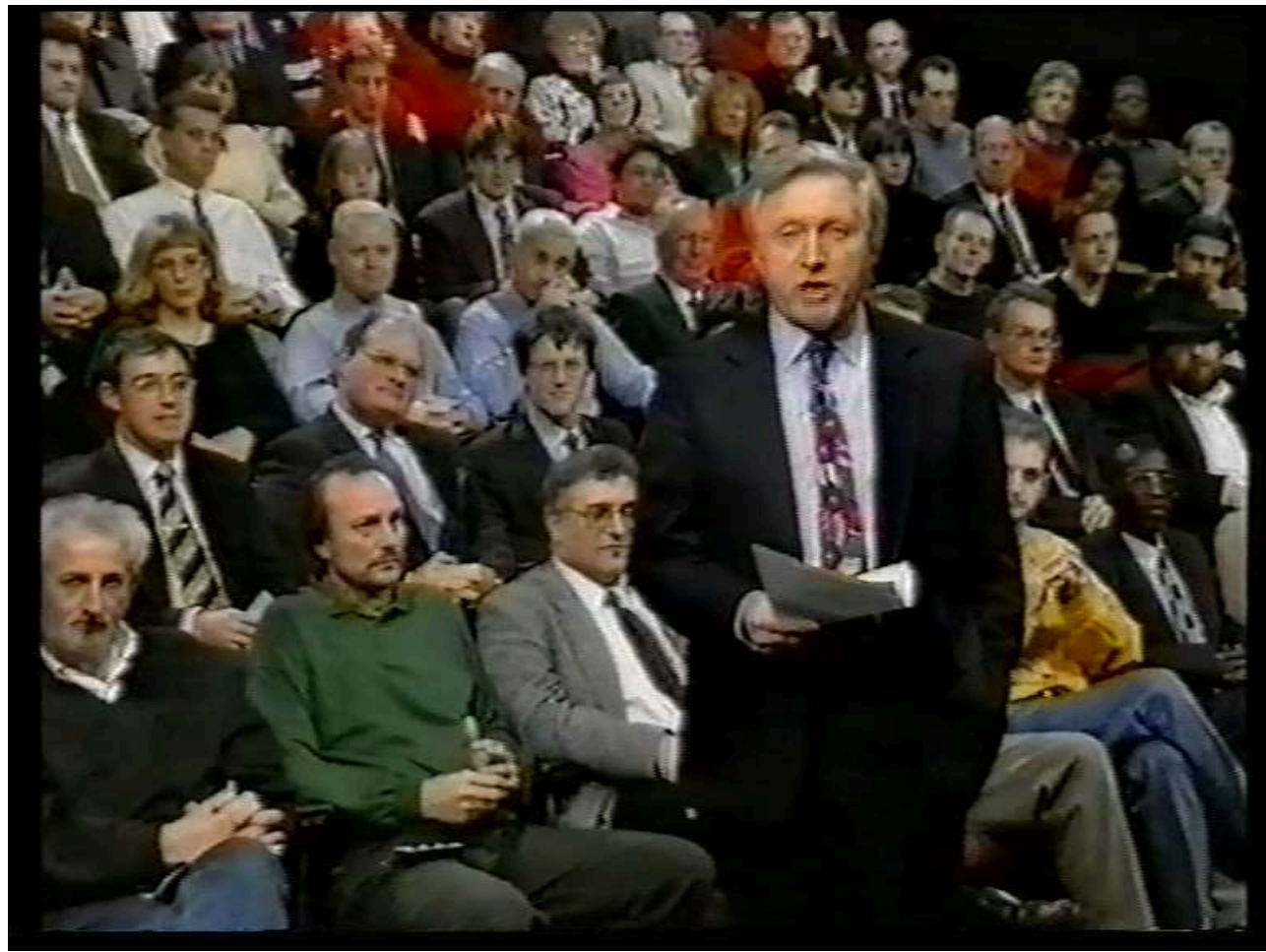

Au cours du débat, il ne trône pas au centre mais se place en bout de table, un peu isolé, comme s'il matérialisait un sas, un relai nécessaire entre le public et les invités.

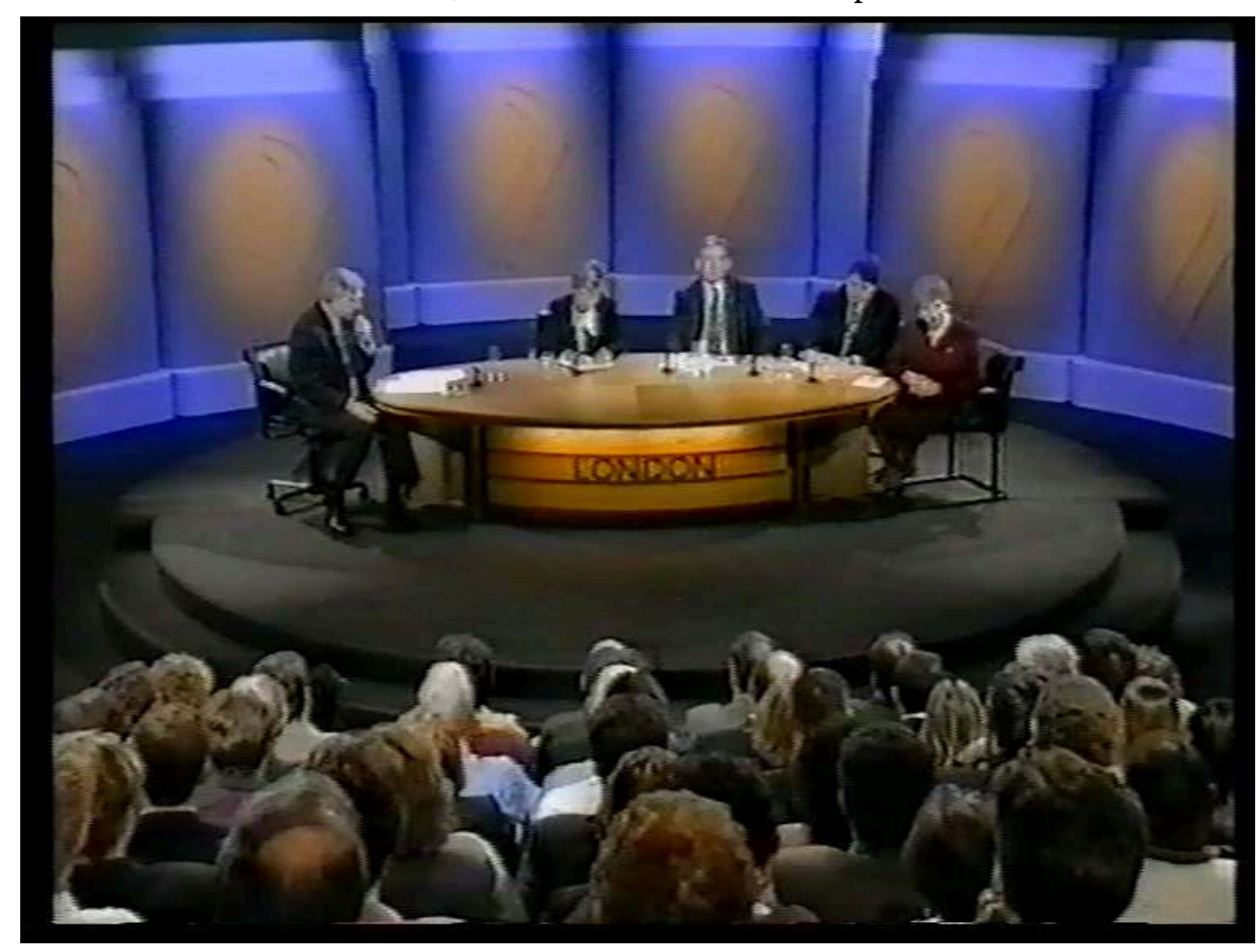




\subsection{Un miroir déformant?}

77 Nous avons voulu tenter une ultime opération statistique pour finaliser l'analyse. Le choc a été grand; si l'on mesure la quantité de discours produite par le public dans l'émission des deux journalistes, on aboutit aux résultats suivants :

Tableau $n^{\circ} 10$. La participation du public quantifiée

\begin{tabular}{|l|l|}
\hline $\begin{array}{l}\text { Interaction } 1 \\
\text { Présentateur Peter Sissons }\end{array}$ & $\begin{array}{l}\text { Interaction 2 } \\
\text { Présentateur David Dimbleby }\end{array}$ \\
\hline $\begin{array}{l}\text { Participation du Public : } \\
\text { Tokens : } 1514\end{array}$ & $\begin{array}{l}\text { Participation du public : } \\
\text { Tokens : } 1302\end{array}$ \\
\hline
\end{tabular}

78 Les chiffres sont sans appel, même si tous les voyants sont au vert pour le public dans la façon de gérer le débat adoptée par Dimbleby, les citoyens ordinaires parlent moins avec Dimbleby que dans l'interaction 1, présentée par le « cassant » Sissons.

79 Life is not always what it seems, pourrait clairement nous venir en tête, et ce, particulièrement pour les médias, dont le rôle n'est pas forcément de « montrer » mais plutôt de "donner à voir », ce qui est bien différent. On fait tout pour montrer que le citoyen ordinaire est une priorité, mais en réalité ce n'est qu'une apparence, sa parole est objectivement réduite.

80 Nous partirons du principe que cet état de fait est cohérent avec le contexte sociopolitique du moment. La deuxième émission datant de mars 1997 est diffusée quelques semaines avant l'élection de Tony Blair et la victoire du « New Labour». Pour les tenants de la CDA (Critical Discourse Analysis, Fairclough: 1995), un événement médiatique est toujours à mettre en relation avec une pratique discursive (en lien avec les dynamiques de consommation $\mathrm{du}$ " produit ») et la pratique socioculturelle (ayant trait avec le contexte large, institutionnel). On est alors en droit de se poser la question suivante : l'avènement $\mathrm{du}$ "New Labour » est-il véritablement un changement de politique, passant du conservatisme au socio-libéral, ou bien simplement un changement d'image, d'affichage ? (Fairclough, 2000). Donne-t-on vraiment la parole au citoyen, ou bien se contente-t-on de lancer des signaux le lui faisant croire?

\section{Conclusion générale}

81 Un certain nombre d'éléments concernant le discours de deux journalistes modérateurs de débats télévisés ont pu être mis au jour dans notre étude. On retiendra que ces journalistes particuliers sont "absents » sur le plan des opinions (le mode de l'unicité est faiblement représenté), mais tout de même bien présents, imprimant un tour particulier au programme qu'ils façonnent au gré de leurs interventions bien souvent méta-discursives (nombre important de gloses). Tour à tour dans la circulation nécessaire de la parole (les appellatifs abondent), le duel stimulant (le trait diaphonique définit leur discours), le relai d'informations contextuelles (genre de l'exposition), ils doivent jouer une partition complexe dont la dimension essentielle et définitoire est diaphonique. 
82 précision dans les outils ainsi qu'une certaine distanciation. Quels que soient les partipris méthodologiques, les orientations qualitative ou quantitative adoptées, il faut garder à l'esprit que les outils (notamment statistiques) sont générateurs de biais et ne sont finalement que des artefacts. Il paraît donc indispensable d'articuler les deux perspectives (qualitative et quantitative) de façon à transcender les limites de chacune par l'éclairage de l'autre, tout en développant une distance critique appropriée vis à vis de l'objet d'étude et des données produites. Un va-et-vient constant est obligatoire entre outils, concepts d'analyse et corpus. De même, il est indispensable d'analyser les discours en prenant en compte le sujet dans toutes ses dimensions, ses positionnements vis à vis du monde et de son environnement, dans une perspective ontologiquement phénoménologique.

\section{BIBLIOGRAPHIE}

Adam, J-M. (1990). Eléments de Linguistique Textuelle. Théorie et Pratique de l'Analyse Textuelle. Mardaga.

Adam, J.M. (1992). Les textes : types et prototypes. Paris : Nathan.

Anthony, L. (2019). AntConc (open source). <https://antconc.en.lo4d.com/windows>

Fairclough, N. (1995). Media Discourse. London: Arnold.

Fairclough, N. (1998). "Political Discourse in the Media: An Analytical Framework", in A. Bell P. Garrett (éds), Approaches to Media Discourse, pp. 142-162. Oxford: Blackwell.

Fairclough, N. (2000). New Labour, New Language? London: Routledge.

Kerbrat-Orecchioni, C. (1980). L'énonciation: De la subjectivité dans le langage. Paris : Armand Colin.

Kerbrat-Orecchioni, C. (1990). Les interactions verbales : Tome I. Paris : Armand Colin.

Kerbrat-Orecchioni, C (1992). Les interactions verbales : Tome II. Paris : Armand Colin.

Ghiglione, R. (ed) (1989). Je vous ai compris ou l'analyse des discours politiques. Paris : Armand Colin.

Livingstone, S., Lunt, P. (1994). Talk on Television, Audience Participation and Public Debate. London : Routledge.

Mayaffre, D., (2005). « Analyse du discours politique et logométrie, point de vue pratique et théorique ", in Langage et Société, n¹14, pp. 91-111.

Oakes, M.P., (1998). Statistics for corpus linguistics. Edimburgh: Edinburgh University Press.

O’Donnell, M., (2002). Systemic Coder (open source) <www.wagsoft.com/coder>

O’Donnell, M., (2012). UAM Corpus Tools (open source) <www.corpustool.com/>

Pourtois, JP., Desmet, H. (1997). Instrumentation et épistémologie en sciences humaines. Bruxelles : ed. Mardaga. 
Rouveyrol, L. (2003a). Etude pragmatique de la variation linguistique dans le débat politique médiatisé en anglais. Thèse N. R., F.Dubois-Charlier (dir), Aix-en-Provence.

Rouveyrol, L. (2003b). « Aspects linguistique du rôle de journaliste dans le débat-panel britannique ", Actes du VIII ${ }^{\text {ème }}$ Colloque International de Communication Sociale (20-24 janvier 2003, Universidad de Santiago de Cuba), L. Miyares (ed), Vol II, pp.731-736.

Rouveyrol, L., Bendinelli, M. (2011). « Parler avec les uns contre les autres, le parallélisme énonciatif comme fait de style du discours politique médiatisé ", in J. Percebois \& M. Petit (éds), Etudes de stylistique anglaise ${ }^{\circ} 2, \mathrm{BSSA}^{\circ} 35$, pp.137-158. Paris : Université de Paris-Ouest Nanterre.

Rouveyrol, L. (2018). « De l'accommodation divergente à la « non-accommodation » dans le débat politique Question Time : essai de modélisation », Anglophonia n ${ }^{\circ} 25 .<$ https://doi.org/10.4000/ anglophonia.1314>. Toulouse : Presses Universitaires du Midi.

Smith, C.S. (2003). Modes of discourse, the local structure of texts. Cambridge: C.U.P.

Vion, R. (1995). « La gestion pluridimensionnelle du dialogue ». in Cahiers de linguistique française $\mathrm{n}^{\circ} 17, \mathrm{pp} .179-203$. Genève : Université de Genève.

Vion, R. (1998). « La mise en scène énonciative du discours », in B. Caron (ed), Proceedings of the 16th international Congress of Linguists, [CD-ROM]. Oxford : Elsevier Sciences.

\section{NOTES}

1. Pour de plus amples informations concernant la nature du débat lui-même, voir Rouveyrol 2018

2. Les chiffres correspondent au nombre de mots au total, les termes d'occurrence et de token sont parfois utilisés.

3. http://www.corpustool.com/

4. Pour un développement plus approfondi du modèle, voir Rouveyrol (2005)

\section{RÉSUMÉS}

Cet article a pour but d'étudier le discours des journalistes modérateurs du débat télévisé Question Time. Il peut sembler a priori inintéressant de se pencher sur la production discursive de participants dont le rôle premier n'est pas de parler mais de «faire parler ». Pourtant, si l'on considère que ces acteurs du débat nécessairement effacés doivent articuler différents ordres de discours (politique, médiatique et celui de la vie ordinaire), il est fascinant de tenter de voir quels positionnements ils réalisent : sont-ils les relais discursifs du citoyen ordinaire ou bien penchentils du côté institutionnel ? Telle est la question qui nous guide dans cette étude. Une approche à la fois qualitative considérant les genres de discours, les positionnements énonciatifs, et quantitative (logométrique) permettant de relativiser les stratégies qualitatives est utilisée. L'analyse révèle l'extrême diversité, pour ne pas dire l'ambiguïté du rôle de modérateur (circulation de la parole, gestion des séquences thématiques). Les variations auxquelles se livrent les deux présentateurs étudiés (Peter Sissons en 1992 et David Dimblebly en 1997) montrent que 
le mode de l'opposition joue un rôle central. Il est aussi suggéré que le contexte socioculturel et politique large doit être pris en compte pour l'interprétation des résultats.

This article aims at studying the discourse of journalists moderating the political debate Question Time. Focusing on the discourse of participants whose role is not to speak but to stimulate the debate may seem pointless. Journalists, however, have to articulate 3 orders of discourse (political, media, ordinary life). It is therefore fascinating to try to identify what positioning is achieved. Do moderators side with ordinary citizens whose discourse they relay or with the Institution? Such is the question guiding the study. The adopted approach is both qualitative, considering discourse genres, enunciative positionings as well as quantitative (logometric), enabling a relative quantification of the strategies at work. The analysis reveals the extreme diversity, not to say ambiguity inherent to the role of debate moderator (articulation of turns, handling of topical sequences). The two moderators scrutinized (Peter Sissons, 1992 and David Dimbleby 1997) show that the mode of the opposition plays a key role to determine variations in terms of strategy. It is also argued that the wider socio-cultural, political context is to be considered in the interpretation of results.

INDEX

Mots-clés : débat politique, modérateur, logométrie, stratégies de positionnement

Keywords : political debate, moderator, logometry, positioning strategies

\section{AUTEUR}

\section{LAURENT ROUVEYROL}

Université Côte d'Azur, UMR 7320 Bases Corpus Langage (Logométrie / Enonciation) 